\title{
MULTIGRID METHODS FOR DISCRETE FRACTIONAL SOBOLEV SPACES*
}

\author{
TRYGVE BæRLAND ${ }^{\dagger}$, MIROSLAV KUCHTA ${ }^{\dagger}$, AND KENT-ANDRE MARDAL $^{\dagger}$
}

\begin{abstract}
Coupled multiphysics problems often give rise to interface conditions naturally formulated in fractional Sobolev spaces. Here, both positive and negative fractionality are common. When designing efficient solvers for discretizations of such problems it would therefore be useful to have a preconditioner for the fractional Laplacian. In this work, we develop an additive multigrid preconditioner for the fractional Laplacian with positive fractionality and show a uniform bound on the condition number. For the case of negative fractionality, we reuse the preconditioner developed for the positive fractionality and left-right multiply a regular Laplacian with a preconditioner with positive fractionality to obtain the desired negative fractionality. Implementational issues are outlined in detail as the differences between the discrete operators and their corresponding matrices must be addressed when realizing these algorithms in code. We finish with some numerical experiments verifying the theoretical findings.
\end{abstract}

Key words. fractional Laplacian, multilevel algorithms, operator preconditioning

AMS subject classifications. $65 \mathrm{M} 60,65 \mathrm{~F} 08,65 \mathrm{~F} 10$

DOI. $10.1137 / 18 \mathrm{M} 1191488$

1. Introduction. Multiphysics or multiscale problems often involve coupling conditions at interfaces which are manifolds of lower dimensions. The coupling conditions, because of the lower dimensionality, are naturally posed in fractional Sobolev spaces, and this fact seemingly complicates discretization schemes and solution algorithms. Our focus here will be on the development of solution algorithms in terms of multilevel preconditioners that from an implementational point of view only require minor adjustments of standard multilevel algorithms.

As simplified examples of problems involving interface conditions, let us consider the following two prototype problems. The first is an elliptic problem with a trace constraint,

$$
\begin{aligned}
-\Delta u+T^{*} \lambda & =f, \quad x \in \Omega, \\
T u & =g, \quad x \in \Gamma,
\end{aligned}
$$

and the second is an elliptic problem in mixed form with a trace constraint,

$$
\begin{array}{rlrl}
u-\nabla p+T^{*} \lambda & =f, & x \in \Omega, \\
\nabla \cdot u & =g, & & x \in \Omega, \\
T u & =h, & & x \in \Gamma .
\end{array}
$$

Here, $\Gamma$ is a submanifold either within $\Omega$ or at its boundary, $T$ is a trace operator, and $T^{*}$ is its adjoint. Both problems are assumed to be equipped with suitable boundary

\footnotetext{
* Submitted to the journal's Methods and Algorithms for Scientific Computing section May 31, 2018; accepted for publication (in revised form) January 24, 2019; published electronically April 2, 2019.

http://www.siam.org/journals/sisc/41-2/M119148.html

Funding: This work was supported by the European Research Council under the European Union's Seventh Framework Programme (FP7/2007-2013) / ERC grant agreement 339643. The second author's work was supported by the Research Council of Norway through grant "RigSpray" (grant 421160).

${ }^{\dagger}$ Department of Mathematics, University of Oslo, Blindern, Oslo, 0316 Norway (trygveba@ math.uio.no,mirok@math.uio.no, kent-and@math.uio.no).
} 
conditions. We remark that although these problems are single physics problems, they may easily be coupled to other problems through the Lagrange multiplier at the interface. As such, the problems represent well the challenge of handling the interface properly in a multiphysics setting.

We may write the above problems as

$$
\left(\begin{array}{cc}
-\Delta & T^{*} \\
T & 0
\end{array}\right)\left(\begin{array}{l}
u \\
\lambda
\end{array}\right)=\left(\begin{array}{l}
f \\
g
\end{array}\right) \text { and } \quad\left(\begin{array}{ccc}
I & -\nabla & T^{*} \\
\nabla \cdot & 0 & 0 \\
T & 0 & 0
\end{array}\right)\left(\begin{array}{l}
u \\
p \\
\lambda
\end{array}\right)=\left(\begin{array}{l}
f \\
g \\
h
\end{array}\right)
$$

A crucial challenge is to discretize and solve these problems in a scalable way such that the computations scale linearly with the number of unknowns. Our approach here is to consider iterative methods and develop preconditioners that are both spectrally equivalent with the involved operators and of order-optimal complexity. The main difficulty is the handling of the Lagrange multiplier, which falls outside the scope of standard multilevel methods. To provide a general framework, we will consider preconditioners constructed in terms of the so-called operator preconditioning approach [32] to be used for iterative methods. As will be explained later, the block diagonal preconditioners constructed by this technique will be of the following form:

$$
\left(\begin{array}{cc}
-\Delta^{-1} & 0 \\
0 & (-\Delta)^{\frac{1}{2}}
\end{array}\right) \quad \text { and } \quad\left(\begin{array}{ccc}
(I-\nabla \nabla \cdot)^{-1} & 0 & 0 \\
0 & I & 0 \\
0 & 0 & (-\Delta)^{-\frac{1}{2}}
\end{array}\right)
$$

respectively. Multilevel methods spectrally equivalent with both $(-\Delta)^{-1}$ and $(I-$ $\nabla \nabla \cdot)^{-1}$ are well known. The challenging part in both cases is the construction of efficient preconditioning algorithms that approximate the inverse of the fractional Laplace problems of the form

$$
(-\Delta)^{s} u=f, \quad x \in \Gamma,
$$

with $s=1 / 2$ and $s=-1 / 2$, equipped with suitable boundary conditions. Furthermore, if $\Gamma$ is of codimension 2, numerical simulations [26] indicate that $s \in(-0.2,-0.1)$ gives rise to efficient preconditioners. In this paper we therefore consider methods for $s \in[-1,1]$.

There are many examples of applications of fractional Laplacians in the literature, and we mention a few that motivate this work. Nonoverlapping domain decomposition preconditioners are studied in $[3,25]$. Here, they use (1.3) with $s=\frac{1}{2}$ to precondition the interface problem involving the related Steklov-Poincaré operator. In [27] the authors use (1.3) with $s=-\frac{1}{2}$ as part of a block diagonal preconditioner for a multiphysics problem where the constraint coupling two domains of different topological dimension is enforced by the Lagrange multiplier. Therein the fractionality $s$ is dictated by the mapping properties of the Schur complement operator. Some further examples of coupled systems with domains of different dimensionality include Babuška's problem for enforcing Dirichlet boundary conditions on an elliptic operator [5], flow stabilization by removal of tangential velocity at the boundary through Lagrange multipliers [8], the no-slip condition on the surface of a falling solid in the Navier-Stokes fluid [17], the inextensibility constraint in the complex model of vesicle formation [1], and the potential jump on a membrane of a cardiac cell [35]. We note that in these applications the fractional Laplace problem has to be solved with both positive and negative exponents. 
There are several alternative approaches that have been used in order to approximate fractional Laplacians. Polynomial approximations of $A^{s}$, where $A$ is a discrete Laplacian, can be computed with standard Krylov subspace methods. However, without any preconditioner a Krylov subspace of large dimension is required for convergence; see, e.g., the Lanczos method in [24, section 4]. Preconditioners based on fractional powers of $A$ on preconditioned problems on small subspaces have been shown to be efficient for various applications in [3, 38]. The contour integral method of [19] and the extended Krylov method of [24] are here related to rational function approximations of $A^{s}$, while [22] considers the best uniform rational approximations of the trasformed function $A \mapsto A^{\beta-s}$. In general, the approximation properties of these methods depend on the condition number of $A$, and thus computations of extremal eigenvalues are often part of the algorithm. Further, the computational complexity of the methods based on rational approximations depends on efficient solvers for auxiliary linear systems, e.g., $\left(A-q_{k} I\right) x=b$ in [22], where $q_{k} \in \mathbb{R}$ is a shift parameter. Almost mesh independent preconditioners for systems arising in [19] and [24] are discussed in [16]. An alternative approach to the matrix transfer method is presented in [9], where the inverse of the fractional Laplacian is defined via the (integral) Balakrishnan formula [6].

Multilevel methods for fractional Laplacians have been considered in $[12,22,33$, 34], but there seems to be a significant untapped potential for advancement. Our work here is closely related to [12], where order-optimal preconditioners for $A^{s}$ when $s \in\left(-\frac{3}{2}, \frac{3}{2}\right)$ were constructed using a hierarchical basis approach. The paper [12], however, only considers smoothers based on level-dependent scaling and does not put much focus on the actual implementation. Here, we will develop and analyze a multilevel algorithm that is straightforward to implement in a standard multilevel software framework. In fact, the main change required is an adjustment of the smoothers. To illustrate the change, let us assume that we want to solve the system $A x=b$, where $A$ is a stiffness matrix corresponding to a discretized Laplacian. A standard Jacobi algorithm can then be written

$$
\mathrm{x}_{i}^{n+1}=\mathrm{x}_{i}^{n}-\frac{1}{\mathrm{~A}_{i, i}} \mathrm{r}_{i}^{n},
$$

where $\mathrm{A}_{i, i}$ are the diagonal entries of the stiffness matrix for a discretized Laplacian, and $r^{n}$ is the residual of the $n$th iterate, $x^{n}$. In our case, for $A^{s} \mathrm{x}=\mathrm{b}$, the proposed Jacobi smoother may be implemented as

$$
\mathrm{x}_{i}^{n+1}=\mathrm{x}_{i}^{n}-\left(\frac{1}{\mathrm{M}_{i, i}^{1-s} \mathrm{~A}_{i, i}^{s}}\right) \mathrm{r}_{i}^{n} .
$$

Here, $\mathrm{M}_{i, i}$ are the diagonal entries of the mass matrix. We notice here that for $s=0$ the action is a Jacobi iteration on the mass matrix, and for $s=1$ the action is a Jacobi iteration on the stiffness matrix, and for $0<s<1$ the action is an interpolation between these two extremes. From an implementational point of view, the restriction and interpolation operators used are the same as those used in standard multilevel algorithms. However, from a theoretical point of view, the fact that we use standard restriction and interpolation operators means that the multilevel approach will be nonnested. In fact, the matrices on coarser levels do not correspond to $(-\Delta)^{s}$-Galerkin projections of the matrix on the finer levels. We therefore employ the framework of nonnested multilevel methods [15]. Furthermore, a multiplicative multilevel algorithm would require computing the residual and hence the evaluation of the exact $(-\Delta)^{-s}$ 
operator on every level. Since the evaluation of the exact $(-\Delta)^{-s}$ is a computationally expensive procedure, we instead rely on the additive multilevel algorithm proposed in [14], where the same residual is used on all levels. The additive variant is significantly less efficient than corresponding multiplicative variants in terms of the conditioning (in the sense that the conditioning depends on the number of levels). Still, this is a small price to pay (only logarithmic in the number of unknowns) to avoid exact evaluation of the residual. In this paper we will assume a quasi-uniform mesh and continuous piecewise linear finite elements. This is mainly for simplicity, and the results can be generalized to higher order discretizations as well as discontinuous Galerkin methods.

The paper is structured as follows. In section 2, we introduce notation and some useful operator inequalities related to fractional powers of positive operators. We also give a brief discussion of fractional Sobolev spaces. Section 3 is devoted to the analysis of an abstract multilevel framework. In section 4 we use this framework to define operators that are spectrally equivalent to the inverse of the fractional Laplacian when the fractionality $s \geq 0$. We discuss some strategies for preconditioning when $s<0$ in section 5 , and in section 6 we discuss implementation of the preconditioners developed in the previous sections. Finally, we provide numerical results that verify our theoretical result in section 7 .

2. Notation and preliminaries. Let $\Omega$ be a bounded, Lipschitz domain in $\mathbb{R}^{n}$, with boundary $\partial \Omega$. We denote by $L^{2}(\Omega)$ the space of square integrable functions over $\Omega$, with inner product $(\cdot, \cdot)$ and norm $\|\cdot\|$. For $k \in \mathbb{N}$, we denote by $H^{k}(\Omega)$ the usual Sobolev spaces of functions in $L^{2}(\Omega)$ with all derivatives up to order $k$ in $L^{2}(\Omega)$. The norm and inner product in $H^{k}$ are denoted by $\|\cdot\|_{k}$ and $(\cdot, \cdot)_{k}$, respectively. The closure in $H^{k}$ of smooth functions with compact support in $\Omega$ is denoted as $H_{0}^{k}(\Omega)$ and its dual space is $H^{-k}$. In general a Hilbert space $X$ is equipped with a norm $\|\cdot\|_{X}$ and an inner product $(\cdot, \cdot)_{X}$, and the dual space is denoted by $X^{\prime}$. For two Hilbert spaces $X$ and $Y$, we write $\mathcal{L}(X, Y)$ to mean the space of bounded linear operators $T: X \rightarrow Y$, which we equip with the usual operator norm

$$
\|T\|_{\mathcal{L}(X, Y)}=\sup _{x \in X} \frac{\|T x\|_{Y}}{\|x\|_{X}} .
$$

Let $A$ be a symmetric positive-definite operator on a finite-dimensional Hilbert space $X$ with dimension $N$. Denote by $\left\{\left(\lambda_{k}, \phi_{k}\right)\right\}_{k=1}^{N}$ the set of eigenpairs of $A$, normalized so that

$$
\left(\phi_{k}, \phi_{l}\right)_{X}=\delta_{k, l},
$$

where $\delta_{k, l}$ is the Kronecker delta. Then $\phi_{k}$ for $k=1, \ldots, N$ forms an orthonormal basis of $X$, and if $u \in X$ has the representation $u=\sum_{k=1}^{N} c_{k} \phi_{k}$, then

$$
A u=\sum_{k=1}^{N} \lambda_{k} c_{k} \phi_{k}
$$

For $s \in \mathbb{R}$, we define the fractional power $A^{s}$ of $A$ by

$$
A^{s} u=\sum_{k=1}^{N} \lambda_{k}^{s} c_{k} \phi_{k} .
$$

If $A$ is only positive semidefinite, then we must restrict to $s \geq 0$, and the eigenvectors corresponding to the nullspace of $A$ are left out (also for $s=0$ ). If $B$ is another 
symmetric positive semidefinite operator on $X$, we write $A \leq B$ if for every $u \in X$

$$
(A u, u)_{X} \leq(B u, u)_{X}
$$

holds. Note that $0 \leq A$ is equivalent to saying that $A$ is positive semidefinite.

A result in operator theory is the Löwner-Heinz inequality, which states that if $A \leq B$, then

$$
A^{s} \leq B^{s}, \quad s \in[0,1]
$$

cf., for instance, [23]. Inequality (2.1) means that the function $x^{s}$ with $x \in[0, \infty)$ is operator monotone for $s \in[0,1]$. It follows that $-(x)^{s}$ is operator convex (cf. [20]); that is, for any two symmetric positive semidefinite operators $A$ and $B$ on a Hilbert space $X$, the inequality

$$
\lambda A^{s}+(1-\lambda) B^{s} \leq(\lambda A+(1-\lambda) B)^{s}
$$

holds for every $\lambda \in[0,1]$. A key result regarding operator convex functions is the Jensen operator inequality (cf. [21, Theorem 2.1]). The version we will use in the current work states that for any $K \in \mathbb{N}$ and $s \in[0,1]$

$$
\sum_{k=1}^{K} P_{k}^{*} A_{k}^{s} P_{k} \leq\left(\sum_{k=1}^{K} P_{k}^{*} A_{k} P_{k}\right)^{s}
$$

where, for $k=1, \ldots, K, A_{k}$ are symmetric positive semidefinite operators on $X$, and $P_{k}$ are linear operators on $X$ so that $\sum_{k=1}^{K} P_{k}^{*} P_{k} \leq I$ and $I$ is the identity operator on $X$.

2.1. Fractional Sobolev spaces. We consider the interpolation spaces between $H^{1}(\Omega)$ and $L^{2}(\Omega)$ as defined in [30]. Let the inner product on $H^{1}(\Omega)$ be realized by the operator $A:=I-\Delta$ as

$$
(u, v)_{1}=(A u, v)=(u, v)+(\nabla u, \nabla v), \quad u, v \in H^{1}(\Omega) .
$$

$A$ is unbounded as an operator mapping $L^{2}(\Omega)$ to $L^{2}(\Omega)$. However, $A$ is well defined on the set

$$
D(A)=\left\{u \in L^{2}(\Omega): A u \in L^{2}(\Omega)\right\},
$$

which is a dense subspace of $L^{2}(\Omega)$. On $D(A), A$ is symmetric and positive-definite, and so the fractional powers of $A, A^{\theta}$ for $\theta \in \mathbb{R}$ are well defined. Note that in the particular case $\theta=\frac{1}{2}$,

$$
\left\|A^{\frac{1}{2}} u\right\|^{2}=(A u, u)=\|u\|_{1} .
$$

For $s \in[0,1]$, we define the fractional Sobolev spaces as

$$
H^{s}(\Omega)=\left\{u \in L^{2}(\Omega): A^{\frac{s}{2}} u \in L^{2}(\Omega)\right\},
$$

which is a Hilbert space with inner product given by

$$
(u, v)_{s}=\left(A^{s} u, v\right), \quad u, v \in H^{s}(\Omega),
$$

and we denote the corresponding norm by $\|\cdot\|_{s}$. 
We define $H_{0}^{s}(\Omega)$ to be the closure of $C_{0}^{\infty}(\Omega)$, the space of infinitely smooth functions with compact support in $\Omega$, in the norm of $H^{s}(\Omega)$. We note that if $s \leq \frac{1}{2}$, the spaces $H_{0}^{s}(\Omega)$ and $H^{s}(\Omega)$ coincide (cf. [30, Theorem 11.1]). For $s \in[-1,0]$, we define a family of fractional Sobolev spaces using the dual of $H_{0}^{s}(\Omega)$. That is,

$$
H^{s}(\Omega)=\left(H_{0}^{-s}(\Omega)\right)^{\prime} .
$$

Replacing $H^{1}(\Omega)$ with $H_{0}^{1}(\Omega)$ and setting $A=-\Delta$ in the above construction will again yield the space $H_{0}^{s}(\Omega)$, with equivalent norm, for all $s$ except when $s=\frac{1}{2}$. In this case, interpolation between $H_{0}^{1}(\Omega)$ and $L^{2}(\Omega)$ results in a space that is strictly contained in $H_{0}^{\frac{1}{2}}(\Omega)$. The subsequent analysis is valid for both $H_{0}^{s}(\Omega)$ and $H^{s}(\Omega)$.

We remark that the above-defined fractional space $H^{s}(\Omega)$ is equivalent to the fractional space $\hat{H}^{s}(\Omega)$ defined in terms of the norm

$$
\|u\|_{\hat{H}^{s}(\Omega)}^{2}=\|u\|^{2}+\int_{\Omega \times \Omega} \frac{|u(x)-u(y)|^{2}}{|x-y|^{n+2 s}} \mathrm{~d} x \mathrm{~d} y .
$$

A detailed overview of the various definitions of fractional Sobolev norms and their discretizations can be found in [31].

2.2. Discrete fractional Sobolev spaces. We will now consider a discretization of the fractional Sobolev spaces $H_{0}^{s}(\Omega)$ and $H^{-s}(\Omega)$ for $s \in[0,1]$. Let $X_{h}$ be a finite-dimensional subspace of $H_{0}^{1}(\Omega)$, with $\operatorname{dim} X_{h}=N_{h}$. We define the operator $A_{h}: X_{h} \rightarrow X_{h}$ by

$$
\left(A_{h} u, v\right)=(\nabla u, \nabla v), \quad u, v \in X_{h} .
$$

Using the fractional powers of $A_{h}$, we define for $s \in \mathbb{R}$ the discrete fractional inner product on $X_{h}$ by

$$
(u, v)_{s, h}=\left(A_{h}^{s} u, v\right), \quad u, v \in X_{h},
$$

and denote the corresponding norm by $\|\cdot\|_{s, h}$. It is clear that for $s=0$ and $s=1$, the two norms $\|\cdot\|_{s, h}$ and $\|\cdot\|_{s}$ coincide on $X_{h}$. Therefore, due to [4, Lemma 2.3], the norms $\|\cdot\|_{s, h}$ and $\|\cdot\|_{s}$, when $s \in[0,1]$, are equivalent on $X_{h}$, with constants of equivalence independent of $N_{h}$.

Let $X_{H}$ be a subspace of $X_{h}$, and let $A_{H}: X_{H} \rightarrow X_{H}$ be defined analogously to $A_{h}$ in (2.4). If $I_{H}: X_{H} \rightarrow X_{h}$ is the inclusion map, we see that

$$
A_{H}=I_{H}^{*} A_{h} I_{H},
$$

where $I_{H}^{*}$ is the adjoint of $I_{H}$ with respect to the $L^{2}$ inner product.

We may also define $A_{H}^{s}: X_{H} \rightarrow X_{H}$, but generally, $A_{H}^{s} \neq I_{H}^{*} A_{h}^{s} I_{H}$. However, by Jensen's operator inequality we have the following.

Lemma 2.1. For every $s \in[0,1]$ we have

$$
I_{H}^{*} A_{h}^{s} I_{H} \leq A_{H}^{s} .
$$

That is, for every $u \in X_{H}$,

$$
\left(A_{h}^{s} u, u\right) \leq\left(A_{H}^{s} u, u\right)
$$


Proof. For $s=0$ and $s=1,(2.6)$ holds with equality, so let $0<s<1$. We start by noticing that since $I_{H}^{*} I_{H}$ is the identity on $X_{H}$,

$$
A_{H}^{2}=\left(I_{H}^{*} I_{H} A_{H} I_{H}^{*} I_{H}\right)^{2}=I_{H}^{*}\left(I_{H} A_{H} I_{H}^{*}\right)^{2} I_{H} .
$$

By induction, we find that

$$
A_{H}^{k}=I_{H}^{*}\left(I_{H} A_{H} I_{H}^{*}\right)^{k} I_{H}
$$

for every nonnegative integer $k$. It follows that for any polynomial $p$

$$
p\left(A_{H}\right)=I_{H}^{*} p\left(I_{H} A_{H} I_{H}^{*}\right) I_{H} .
$$

Take now $\epsilon>0$. The spectra of both $A_{H}$ and $I_{H} A_{H} I_{H}^{*}$ are contained in some bounded, nonnegative interval $[0, b]$. By the Weierstrass approximation theorem we can thus choose a polynomial $p$ so that

$$
\left\|\left(I_{H} A_{H} I_{H}^{*}\right)^{s}-p\left(I_{H} A_{H} I_{H}^{*}\right)\right\|<\epsilon \quad \text { and } \quad\left\|A_{H}^{s}-p\left(A_{H}\right)\right\|<\epsilon .
$$

Using the triangle inequality and (2.7), we have that

$\left\|A_{H}^{s}-I_{H}^{*}\left(I_{H} A_{H} I_{H}^{*}\right)^{s} I_{H}\right\| \leq\left\|A_{H}^{s}-p\left(A_{H}\right)\right\|+\left\|I_{H}^{*}\left(\left(I_{H} A_{H} I_{H}^{*}\right)^{s}-p\left(I_{H} A_{H} I_{H}^{*}\right)\right) I_{H}\right\|<2 \epsilon$, and since $\epsilon$ was arbitrary, this shows that

$$
A_{H}^{s}=I_{H}^{*}\left(I_{H} A_{H} I_{H}^{*}\right)^{s} I_{H} .
$$

Using (2.5) in (2.8), we get that

$$
A_{H}^{s}=I_{H}^{*}\left(I_{H} I_{H}^{*} A_{h} I_{H} I_{H}^{*}\right)^{s} I_{H} .
$$

Finally, $I_{H} I_{H}^{*}$ defines a symmetric operator on $X_{h}$ with $L^{2}$ operator norm equal to 1. Since the function $x \mapsto-x^{s}$ is operator convex on $[0, \infty)$, we can use Jensen's operator inequality $(2.2)$ in (2.9) to get

$$
\begin{aligned}
A_{H}^{s} & \geq I_{H}^{*} I_{H} I_{H}^{*} A_{h}^{s} I_{H} I_{H}^{*} I_{H} \\
& =I_{H}^{*} A_{h}^{s} I_{H},
\end{aligned}
$$

where we have used that $I_{H}^{*} I_{H}$ is the identity on $X_{H}$.

3. Abstract multilevel theory. In order to analyze and implement a multigrid preconditioner for the fractional Laplacian, there are three main issues that need to be dealt with. First, we need to derive and implement a smoother with the desired properties. As already mentioned in the introduction, this step only requires a minor modification to standard smoothing algorithms. We will discuss the details concerning implementation later. Second, the restriction/interpolation operators do not result in a nested hierarchy of operators in our fractional setting as $A_{H}^{s} \neq I_{H}^{*} A_{h}^{s} I_{H}$. For this reason we will employ the framework for nonnested multilevel algorithms developed in [15]. Third, our main motivation for developing fractional multilevel solvers is their application to multiphysics and multiscale problems where the preconditioner for the fractional Laplacian is utilized at the interfaces. As such, the fractional Laplacian operator is not part of the original problem, and therefore we may not assume that this operator has been implemented. Furthermore, implementing this operator in an efficient manner is a challenge but is currently a very active research field; cf., e.g., [31] 
for an overview. To avoid the application of the fractional Laplacian on the various levels we employ additive multilevel schemes which enable the residual of the problem to be used at all levels and remove the need for implementing a global fractional Laplacian operator. That said, the theory developed here extends to multiplicative algorithms for problems involving the fractional Laplacian, such as the standard Vcycle. In this section we will address the second and the third issues and outline a theory for an additive multilevel scheme applied to an abstract nonnested problem. As such, the analysis of this section is a synthesis of the papers [14] and [15].

Assume that we are given a nested sequence of finite-dimensional function spaces

$$
V_{1} \subset V_{2} \subset \cdots \subset V_{J}=V, \quad J \geq 2 .
$$

We further assume that $V$, and consequently all subspaces of $V$, is endowed with an inner product $(\cdot, \cdot)$, with corresponding induced norm $\|\cdot\|$. Moreover, for each $k=1, \ldots, J$, we assume that we are given a symmetric positive-definite operator $A_{k}: V_{k} \rightarrow V_{k}$, and we set $A=A_{J}$. Note that we do not assume that the $A_{k}$ operators are nested.

For the development and analysis of our multilevel algorithm, it will be useful to define a number of operators on each level $k$. First, we define $P_{k, k-1}: V_{k} \rightarrow V_{k-1}$ by

$$
\left(A_{k-1} P_{k, k-1} v, w\right)=\left(A_{k} v, w\right) \quad \forall v \in V_{k}, w \in V_{k-1} .
$$

We remark that in a nested setting, $P_{k, k-1}$ is the $A$-projection, while since the $A_{k}$ operators are not nested, the $P_{k, k-1}$ operators are not projections. Next, we define $Q_{k}: V \rightarrow V_{k}$ by

$$
\left(Q_{k} v, w\right)=(v, w) \quad \forall v \in V, w \in V_{k} .
$$

It follows by the above definitions that

$$
A_{k-1} P_{k, k-1}=Q_{k-1} A_{k}
$$

and $Q_{l} Q_{k}=Q_{k} Q_{l}=Q_{l}$ whenever $l \leq k$. For the sake of brevity, it will also be useful to define $P_{k}: V \rightarrow V_{k}$ by $P_{k}=P_{k+1, k} P_{k+2, k+1} \cdots P_{J, J-1}$. Using the definition of $P_{j+1, j}$ for $j=k, \ldots, J-1$, we see that

$$
\left(A_{k} P_{k} v, w\right)=(A v, w) \quad \forall v \in V, w \in V_{k} .
$$

Furthermore, applying (3.3) repeatedly, we find that

$$
A_{k} P_{k}=Q_{k} A .
$$

Finally, suppose we are given for each $k$ a smoother, which is a symmetric positive definite operator $R_{k}: V_{k} \rightarrow V_{k}$ and in some sense should approximate $A_{k}^{-1}$ on $V_{k} \backslash V_{k-1}$. We can now define an additive multilevel operator $B: V \rightarrow V$ by

$$
B=\sum_{k=1}^{J} R_{k} Q_{k} .
$$

As remarked in [14], $B$ can be viewed as an additive version of the standard multiplicative $\mathrm{V}$-cycle multigrid algorithm, where $R_{k}$ plays the role of smoother. Because of this, it is reasonable that the assumptions we need to make to establish spectral 
equivalence between $A^{-1}$ and $B$ are similar to those made for standard multigrid algorithms.

We assume that for $k=2, \ldots, J$

$$
\left(A_{k} v, v\right) \leq\left(A_{k-1} v, v\right) \quad \forall v \in V_{k-1} .
$$

Under assumption (A.1) and the definition of $P_{k, k-1}$ we see that for any $v \in V_{k}$

$$
\begin{aligned}
\left(A_{k-1} P_{k, k-1} v, P_{k, k-1} v\right) & =\left(A_{k} v, P_{k, k-1} v\right) \\
& \leq\left(A_{k} P_{k, k-1} v, P_{k, k-1} v\right)^{\frac{1}{2}}\left(A_{k} v, v\right)^{\frac{1}{2}} \\
& \leq\left(A_{k-1} P_{k, k-1} v, P_{k, k-1} v\right)^{\frac{1}{2}}\left(A_{k} v, v\right)^{\frac{1}{2}} .
\end{aligned}
$$

Thus, (A.1) implies

$$
\left(A_{k-1} P_{k, k-1} v, P_{k, k-1} v\right) \leq\left(A_{k} v, v\right) \quad \forall v \in V_{k} .
$$

Conversely, assume (3.6). Then, for any $v \in V_{k-1}$, by the definition of $P_{k, k-1}$,

$$
\begin{aligned}
\left(A_{k} v, v\right) & =\left(A_{k-1} P_{k, k-1} v, v\right) \\
& \leq\left(A_{k-1} P_{k, k-1} v, P_{k, k-1} v\right)^{\frac{1}{2}}\left(A_{k-1} v, v\right)^{\frac{1}{2}} \\
& \leq\left(A_{k} v, v\right)^{\frac{1}{2}}\left(A_{k-1} v, v\right)^{\frac{1}{2}}
\end{aligned}
$$

which implies (A.1). Thus, (A.1) and (3.6) are equivalent. Notice that a similar inequality to (3.6) would also hold for $P_{k}$, namely

$$
\left(A_{k} P_{k} v, P_{k} v\right) \leq(A v, v) \quad \forall v \in V .
$$

For the operators $R_{k}$, we assume there are constants $C_{1}, C_{2}>0$ independent of $k$ so that

$$
C_{1} \frac{\|v\|^{2}}{\lambda_{k}} \leq\left(R_{k} v, v\right) \leq C_{2}\left(A_{k}^{-1} v, v\right) \quad \forall v \in V_{k}
$$

where $\lambda_{k}$ is the largest eigenvalue of $A_{k}$. Finally, as is common in multigrid theory, we will use an approximation assumption to establish spectral equivalence between $B$ and $A^{-1}$. In this work, we assume the following approximation property: There are an $\alpha \in(0,1]$ and a constant $C_{3}>0$, independent of $k$, so that

$$
\left(A_{k}\left(I-P_{k, k-1}\right) v, v\right) \leq C_{3}^{\alpha}\left(\frac{\left\|A_{k} v\right\|^{2}}{\lambda_{k}}\right)^{\alpha}\left(A_{k} v, v\right)^{1-\alpha} \quad \forall v \in V_{k} .
$$

We are now in a position to state and prove the main theorem of this section. The proof closely resembles the proofs of Corollary 3 and Theorem 2 in [14] but is extended to handle the case of the nonnestedness of the operators.

Theorem 3.1. Assume that (A.1), (A.2), and (A.3) hold. Then, with B given in $(3.5)$,

$$
C_{1} C_{3}^{-1} J^{1-\frac{1}{\alpha}}(A v, v) \leq(B A v, A v) \leq C_{2} J(A v, v)
$$

holds for every $v \in V$. 
Proof. Fix $v \in V$. Using the definition of $B$ together with (3.4), we find that

$$
(B A v, A v)=\sum_{k=1}^{J}\left(R_{k} Q_{k} A v, Q_{k} A v\right)=\sum_{k=1}^{J}\left(R_{k} A_{k} P_{k} v, A_{k} P_{k} v\right) .
$$

Thus, applying the second inequality of (A.2) and (3.7) gives

$$
(B A v, A v) \leq C_{2} \sum_{k=1}^{J}\left(A_{k} P_{k} v, P_{k} v\right) \leq C_{2} J(A v, v),
$$

which proves the second inequality of (3.8).

For the first inequality of (3.8) we write

$$
v=\sum_{k=1}^{J}\left(P_{k}-P_{k-1}\right) v,
$$

where we interpret $P_{0}=0$ and $P_{J}=I$. By the definition of $P_{k}$, we have that $P_{k-1}=P_{k, k-1} P_{k}$, and so

$$
v=\sum_{k=1}^{J}\left(I-P_{k, k-1}\right) P_{k} v
$$

It follows that

$$
(A v, v)=\sum_{k=1}^{J}\left(A_{k}\left(I-P_{k, k-1}\right) P_{k} v, P_{k} v\right) .
$$

Using (A.3) and (3.7) gives

$$
\begin{aligned}
(A v, v) & \leq C_{3}^{\alpha} \sum_{k=1}^{J}\left(\lambda_{k}^{-1}\left\|A_{k} P_{k} v\right\|^{2}\right)^{\alpha}\left(A_{k} P_{k} v, P_{k} v\right)^{1-\alpha} \\
& \leq C_{3}^{\alpha}(A v, v)^{1-\alpha} \sum_{k=1}^{J}\left(\lambda_{k}^{-1}\left\|A_{k} P_{k} v\right\|^{2}\right)^{\alpha} .
\end{aligned}
$$

The first inequality of (A.2) then implies that

$$
\begin{aligned}
(A v, v) & \leq\left(C_{1}^{-1} C_{3}\right)^{\alpha}(A v, v)^{1-\alpha} \sum_{k=1}^{J}\left(R_{k} A_{k} P_{k} v, A_{k} P_{k} v\right)^{\alpha} \\
& \leq\left(C_{1}^{-1} C_{3}\right)^{\alpha} J^{1-\alpha}(A v, v)^{1-\alpha}\left(\sum_{k=1}^{J}\left(R_{k} A_{k} P_{k} v, A_{k} P_{k} v\right)\right)^{\alpha} \\
& \leq\left(C_{1}^{-1} C_{3}\right)^{\alpha} J^{1-\alpha}(A v, v)^{1-\alpha}(B A v, A v)^{\alpha}
\end{aligned}
$$

where the second step follows by Hölder's inequality. The last step follows by the definition of $B$ in (3.5), and (3.4). Dividing by $\left(C_{1}^{-1} C_{3}\right)^{\alpha}(A v, v)^{1-\alpha} J^{1-\alpha}$ on both sides and raising to the power $\frac{1}{\alpha}$ gives the first inequality of (3.8).

Remark 1. Analogously to what was done in [14], we can replace the regularity assumption (A.3) with an assumption on the projections $Q_{k}$ (cf. also [13]). In particular, if, instead of (A.3), we assume that there is a constant $C_{4}>0$ independent of $k$ so that

$$
\left\|\left(I-Q_{k-1}\right) v\right\|^{2} \leq C_{4} \lambda_{k}^{-1}(A v, v) \quad \forall v \in V,
$$


then we can use an argument like that made in [14, Theorem 1 and Corollary 1] to show that

$$
C_{4}^{-1} C_{1} J^{-1}(A v, v) \leq(B A v, A v) \leq C_{2} J(A v, v)
$$

for every $v \in V$.

4. Preconditioner for discrete fractional Laplacian. In this section we use the abstract theory developed in section 3 to derive an order optimal preconditioner for the discrete fractional Laplacian $A_{h}^{s}$, described in section 2 , when $s \in[0,1]$.

Let $\Omega$ be a bounded, polygonal domain in $\mathbb{R}^{n}$, and suppose we are given a quasiuniform triangulation of $\Omega$, denoted by $\mathcal{T}_{h}$, where $h$ denotes the characteristic mesh size. We restrict our discussion to the case when $V_{h}$ is the space of continuous, piecewise linear functions relative to the triangulation $\mathcal{T}_{h}$ which vanish on $\partial \Omega$. To define a nested sequence of subspaces, we suppose that $\mathcal{T}_{h}$ is constructed by successive refinements. That is, we are given a sequence,

$$
\mathcal{T}_{1} \subset \cdots \mathcal{T}_{J}=\mathcal{T}_{h},
$$

of quasi-uniform triangulations, and $\mathcal{T}_{k}$ has characteristic mesh size $h_{k}$ for $k=$ $1, \ldots, J$. In the following, we will assume the bounded refinement hypothesis, that is, $h_{k-1} \leq \gamma h_{k}$ for $k=2, \ldots, J$, where $\gamma \geq 1$ is a constant. In practice, $\gamma$ is around 2 . For each $k$ we define $V_{k}$ as the space of continuous, piecewise linear functions relative to $\mathcal{T}_{k}$ that vanish on $\partial \Omega$. Further, we define $A_{k}: V_{k} \rightarrow V_{k}$ by

$$
\left(A_{k} v, w\right)=(\nabla v, \nabla w), \quad v, w \in V_{k} .
$$

We now fix $s \in[0,1]$. Since $A_{k}$ is symmetric positive-definite, we can define $A_{k}^{s}$ and corresponding norms

$$
\|v\|_{s, k}^{2}:=\left(A_{k}^{s} v, v\right), \quad v \in V_{k} .
$$

Note that if $s=0$ or $s=1$, the norm $\|\cdot\|_{s, k}$ coincides with the $L^{2}$-norm and $H_{0}^{1}$-norm, respectively. That is, $\|\cdot\|_{0, k}=\|\cdot\|$ and $\|\cdot\|_{1, k}=\|\cdot\|_{1}$.

Analogous to the discussion in section 3 we also define operators $P_{k, k-1}^{s}: V_{k} \rightarrow$ $V_{k-1}$ by

$$
\left(A_{k-1}^{s} P_{k, k-1}^{s} v, w\right)=\left(A_{k}^{s} v, w\right) \quad \forall v \in V_{k}, w \in V_{k-1},
$$

$Q_{k}: V_{J} \rightarrow V_{k}$ is the $L^{2}$-projection, and $P_{k}^{s}:=P_{k+1, k}^{s} P_{k+2, k+1}^{s} \cdots P_{J, J-1}^{s}$.

To complete the description of a multilevel preconditioner, we still need to define smoothers, $R_{k}^{s}$, for each $k$ and $s$. In this work, we will define additive smoothers based on domain decomposition. To that end, let $\mathcal{N}_{k}$ be the set of vertices in the triangulation $\mathcal{T}_{k}$, and for each $\nu \in \mathcal{N}_{k}$, let $\mathcal{T}_{k, \nu}$ be the set of triangles meeting at the vertex $\nu$. Then $\mathcal{T}_{k, \nu}$ forms a triangulation of a small subdomain $\Omega_{k, \nu}$. We define $V_{k, \nu}$ to be the subspace of functions in $V_{k}$ with support contained in $\bar{\Omega}_{k, \nu}$. Analogously to what we did for $V_{k}$, we may define for each $\nu \in \mathcal{N}_{k}$ operators $A_{k, \nu}^{s}: V_{k, \nu} \rightarrow V_{k, \nu}$ and $Q_{k, \nu}: V_{k} \rightarrow V_{k, \nu}$. For $k=2, \ldots, J$, we define

$$
R_{k}^{s}:=\sum_{\nu \in \mathcal{N}_{k}} A_{k, \nu}^{-s} Q_{k, \nu},
$$


while on the coarsest level we set $R_{1}^{s}=A_{1}^{-s}$. We note that the smoothers are symmetric positive-definite, and their inverses satisfy

$$
\left(\left(R_{k}^{s}\right)^{-1} v, v\right)=\inf _{\substack{v=\sum_{\nu} v_{\nu} \in V_{k, \nu} \\ v_{\nu}}} \sum_{\nu \in \mathcal{N}_{k}}\left(A_{k, \nu}^{s} v_{\nu}, v_{\nu}\right), \quad v \in V_{k} .
$$

With our particular choice of subspaces $V_{k, \nu}$, any $v \in V_{k}$ can be uniquely decomposed into $v=\sum_{\nu \in \mathcal{N}_{k}} v_{\nu}$, with $v_{\nu} \in V_{k, \nu}$. Moreover, it is well known that this decomposition is $L^{2}$-stable. That is, there are constants $K_{0}, K_{1}>0$ independent of $k$ and $v$ so that

$$
K_{0}\|v\|^{2} \leq \sum_{\nu \in \mathcal{N}_{k}}\left\|v_{\nu}\right\|^{2} \leq K_{1}\|v\|^{2} .
$$

Our preconditioner now reads

$$
B_{h}^{s}:=\sum_{k=1}^{J} R_{k}^{s} Q_{k} .
$$

We want to apply Theorem 3.1 to the preconditioner defined by (4.5) and (4.2), so we need to verify assumptions (A.1)-(A.3).

Using Lemma 2.1, we immediately find that for every $k$,

$$
\left(A_{k}^{s} v, v\right) \leq\left(A_{k-1}^{s} v, v\right) \quad \forall v \in V_{k-1},
$$

which verifies (A.1) in the current context.

We present the verification of (A.2) in the following lemma.

Lemma 4.1. For $k=1, \ldots, J$, let $R_{k}^{s}: V_{k} \rightarrow V_{k}$ be defined by (4.2). Then there are constants $C_{1}, C_{2}>0$, so that for every $k$,

$$
C_{1} \frac{\|v\|^{2}}{\lambda_{k}^{s}} \leq\left(R_{k}^{s} v, v\right) \leq C_{2}\left(A_{k}^{-s} v, v\right) \quad \forall v \in V_{k},
$$

where $\lambda_{k}^{s}$ is the largest eigenvalue of $A_{k}^{s}$.

Proof. It is evident that (4.6) holds on the coarsest level; i.e., for $k=1$ (4.6) is satisfied with $C_{1}=C_{2}=1$. So let $k \geq 2$, and fix $v \in V_{k}$. For $\nu \in \mathcal{N}_{k}$, let $\lambda_{k, \nu}^{s}$ denote the largest eigenvalue of $A_{k, \nu}^{s}$. To prove the first inequality in (4.6), we begin by noting that

$$
\lambda_{k}^{1}=\sup _{w \in V_{k}} \frac{\left(A_{k}^{1} w, w\right)}{(w, w)} \geq \sup _{w \in V_{k, \nu}} \frac{\left(A_{k, \nu}^{1} w, w\right)}{(w, w)}=\lambda_{k, \nu}^{1} .
$$

Thus, since $\lambda_{k}^{s}=\left(\lambda_{k}^{1}\right)^{s}$, we have that

$$
\lambda_{k}^{s} \geq \lambda_{k, \nu}^{s} .
$$

Let now $v=\sum_{\nu \in \mathcal{N}_{k}} v_{\nu}$ be the unique decomposition of $v$ into $V_{k, \nu}$ for $\nu \in \mathcal{N}_{k}$. Using (4.7) and the second inequality of (4.4), together with the definition of $Q_{k, \nu}$ and $R_{k}^{s}$, 
yields

$$
\begin{aligned}
\frac{(v, v)}{\lambda_{k}^{s}} & =\frac{1}{\lambda_{k}^{s}} \sum_{\nu \in \mathcal{N}_{k}}\left(v, v_{\nu}\right) \\
& =\frac{1}{\lambda_{k}^{s}} \sum_{\nu \in \mathcal{N}_{k}}\left(Q_{k, \nu} v, v_{\nu}\right) \\
& \leq\left(\frac{1}{\lambda_{k}^{s}} \sum_{\nu \in \mathcal{N}_{k}}\left(Q_{k, \nu} v, Q_{k, \nu} v\right)\right)^{\frac{1}{2}}\left(\frac{1}{\lambda_{k}^{s}} \sum_{\nu \in \mathcal{N}_{k}}\left\|v_{\nu}\right\|^{2}\right)^{\frac{1}{2}} \\
& \leq\left(\sum_{\nu \in \mathcal{N}_{k}} \frac{1}{\lambda_{k, \nu}^{s}}\left(Q_{k, \nu} v, Q_{k, \nu} v\right)\right)^{\frac{1}{2}}\left(\frac{K_{1}}{\lambda_{k}^{s}}\|v\|^{2}\right)^{\frac{1}{2}} \\
& \leq\left(\sum_{\nu \in \mathcal{N}_{k}}\left(A_{k, \nu}^{-s} Q_{k, \nu} v, Q_{k, \nu} v\right)\right)^{\frac{1}{2}}\left(\frac{K_{1}}{\lambda_{k}^{s}}\|v\|^{2}\right)^{\frac{1}{2}} \\
& \leq\left(R_{k}^{s} v, v\right)^{\frac{1}{2}}\left(\frac{K_{1}}{\lambda_{k}^{s}}\|v\|^{2}\right)^{\frac{1}{2}},
\end{aligned}
$$

which proves the first inequality of (4.6) with $C_{1}=K_{1}^{-1}$.

For the second inequality, we begin by noting that for $s=1$, it was proven in [37, Lemma 7.2] that there is a constant $C$ independent of $k$ so that

$$
\left(R_{k}^{1} v, v\right) \leq C\left(A_{k}^{-1} v, v\right) \quad \forall v \in V_{k} .
$$

Since $s \in[0,1]$, it follows by the Löwner-Heinz inequality (2.1) that

$$
\left(\left(R_{k}^{1}\right)^{s} v, v\right) \leq C^{s}\left(A_{k}^{-s} v, v\right) \quad \forall v \in V_{k} .
$$

Thus, if we can show that

$$
\left(R_{k}^{s} v, v\right) \leq C\left(\left(R_{k}^{1}\right)^{s} v, v\right)
$$

for some constant $C$, which is independent of $k$, then (4.8) together with (4.9) implies the second inequality of (4.6).

We aim to prove (4.9) using Jensen's operator inequality. To that end, we need to scale $R_{k}^{s}$, so that (2.2) is applicable. From the characterization of $\left(R_{k}^{0}\right)^{-1}$ in (4.3) and the first inequality of (4.4) we have that

$$
K_{0}\|v\|^{2} \leq\left(\left(R_{k}^{0}\right)^{-1} v, v\right),
$$

which in turn implies that

$$
\sum_{\nu \in \mathcal{N}_{k}}\left(Q_{k, \nu} v, Q_{k, \nu} v\right)=\left(R_{k}^{0} v, v\right) \leq K_{0}^{-1}\|v\|^{2} .
$$

If we now define $\tilde{Q}_{k, \nu}=K_{0}^{\frac{1}{2}} Q_{k, \nu}$ and $\tilde{R}_{k}^{s}=K_{0} R_{k}^{s}$, we have that

$$
\sum_{\nu \in \mathcal{N}_{k}}\left(\tilde{Q}_{k, \nu} v, \tilde{Q}_{k, \nu} v\right) \leq\|v\|^{2} \text { and }\left(\tilde{R}_{k}^{s} v, v\right)=\sum_{\nu \in \mathcal{N}_{k}}\left(A_{k, \nu}^{-s} \tilde{Q}_{k, \nu} v, \tilde{Q}_{k, \nu} v\right) .
$$


We can now use Jensen's operator inequality (2.2), together with an argument analogous to that in the proof of Lemma 2.1, to get

$$
\begin{aligned}
R_{k}^{s} & =K_{0}^{-1} \tilde{R}_{k}^{s} \\
& \leq K_{0}^{-1}\left(\tilde{R}_{k}^{1}\right)^{s} \\
& =K_{0}^{-(1-s)}\left(R_{k}^{1}\right)^{s} .
\end{aligned}
$$

This, together with (4.8), proves the second inequality of (4.6) with $C_{2}=K_{0}^{-(1-s)} C^{s}$.

We observe that the proof of Lemma 4.1 shows that if the decomposition $V_{k}=$ $\sum_{\eta \in \mathcal{N}_{k}} V_{k, \nu}$ is stable in both the $L^{2}$-norm and the $H_{0}^{1}$-norm, then it is also stable in the fractional norm $\|\cdot\|_{s, k}$. That is, if there are constants $c_{0}, c_{1}>0$ so that

$$
\left(A_{k}^{s} v, v\right) \leq c_{s}\left(\left(R_{k}^{s}\right)^{-1} v, v\right) \quad \forall v \in V_{k},
$$

with $s=0$ and $s=1$, then the same holds for every $s \in[0,1]$, with $c_{s}=c_{0}^{1-s} c_{1}^{s}$. In this way, the smoother defined by (4.2) is the natural interpolation between the corresponding smoothers for $s=0$ and $s=1$. As such, the results in Lemma 4.1 can be extended to more general overlapping domain decompositions than the one we consider here.

As noted in [11, Remark 5.1], the $\alpha$ in the approximation and regularity assumption (A.3) is closely related to the elliptic regularity of the continuous problem. Therefore, we make the following assumption.

Assumption 4.1. There is an $\alpha^{\prime} \in(0,1]$ so that $A$ is a bounded operator from $H_{0}^{1}(\Omega) \bigcap H^{1+\alpha^{\prime}}(\Omega)$ to $H^{-1+\alpha^{\prime}}(\Omega)$, and $A^{-1}$ is a bounded operator from $H^{-1+\alpha^{\prime}}(\Omega)$ to $H_{0}^{1}(\Omega) \bigcap H^{1+\alpha^{\prime}}(\Omega)$.

Assumption 4.1 is standard for proving condition (A.3) in the case of $s=1$ (cf., for instance, [11]). In [9, Thm 4.3, and Rem. 4.1] Bonito and Pasciak used Assumption 4.1 to prove the error estimate

$$
\left\|\left(A^{-s}-A_{k}^{-s} Q_{k}\right) f\right\| \leq C h_{k}^{2 s}\|f\| \quad \forall f \in L^{2}(\Omega),
$$

when $\alpha^{\prime}>s$. By the triangle inequality and the bounded refinement hypothesis it then follows that

$$
\left\|\left(A_{k}^{-s}-A_{k-1}^{-s} Q_{k-1}\right) f\right\| \leq C h_{k}^{2 s}\|f\| \quad \forall f \in V_{k},
$$

for each $k$. This estimate is sufficient to verify (A.3) in our current context. The result is stated in the following lemma.

Lemma 4.2. Assume that Assumption 4.1 is satisfied with $\alpha^{\prime}>s$. Then there is a constant $C_{3}>0$, so that for every $k$

$$
\left(A_{k}^{s}\left(I-P_{k, k-1}^{s}\right) v, v\right) \leq C_{3} \frac{\left\|A_{k}^{s} v\right\|^{2}}{\lambda_{k}^{s}} .
$$

Proof. From the definition of $P_{k, k-1}^{s}$ in (4.1),

$$
I-P_{k, k-1}^{s}=I-A_{k-1}^{-s} Q_{k-1} A_{k}^{s}=\left(A_{k}^{-s}-A_{k-1}^{-s} Q_{k-1}\right) A_{k}^{s},
$$

and so, for any $v \in V_{k}$

$$
\left(A_{k}^{s}\left(I-P_{k, k-1}^{s}\right) v, v\right) \leq\left(\left(A_{k}^{-s}-A_{k-1}^{-s} Q_{k-1}\right) A_{k}^{s} v, A_{k}^{s} v\right) .
$$


Using the Cauchy-Schwarz inequality together with the error estimate (4.10), we get

$$
\left(A_{k}^{s}\left(I-P_{k, k-1}^{s}\right) v, v\right) \leq\left\|\left(A_{k}^{-s}-A_{k-1}^{-s} Q_{k-1}\right) A_{k}^{s} v\right\|\left\|A_{k}^{s} v\right\| \leq C h_{k}^{2 s}\left\|A_{k}^{s} v\right\|^{2} .
$$

By the quasi-uniformity of the mesh $h_{k}^{2} \leq C \lambda_{k}^{-1}$, and it follows that $h_{k}^{2 s} \leq C \lambda_{k}^{-s}$. Using this in (4.12) completes the proof.

We are finally in a position to prove the main theorem of this section.

TheOREM 4.1. Let Assumption 4.1 be satisfied with $\alpha^{\prime}>s$. Then, for $s \in[0,1]$, $B_{h}^{s}$ defined by (4.2) and (4.5) satisfies

$$
C_{1} C_{3}^{-1}\left(A_{h}^{s} v, v\right) \leq\left(B_{h}^{s} A_{h}^{s} v, A_{h}^{s} v\right) \leq C_{2} J\left(A_{h}^{s} v, v\right) \quad \forall v \in V,
$$

where $C_{1}, C_{2}$, and $C_{3}$ are the same as in Lemmas 4.1 and 4.2 .

Proof. This result is a straightforward application of Theorem 3.1 together with Lemmas 4.1 and 4.2.

Theorem 4.1 shows that the condition number $K\left(B_{h}^{s} A_{h}^{s}\right) \leq C J$ and so increases linearly with the number of mesh levels but is independent of $\bar{h}$.

With less regularity of the domain, we can still prove a slightly weaker form of spectral equivalence. By the assumed quasi-uniformity of $\mathcal{T}_{k}$, we have for $k=2, \ldots, J$ that

$$
\left\|\left(I-Q_{k-1}\right) v\right\|^{2} \leq C h_{k}^{2}\|v\|_{1}^{2} \quad \forall v \in V_{k} .
$$

This, together with the boundedness of $I-Q_{k-1}$ and interpolation theory, yields

$$
\left\|\left(I-Q_{k-1}\right) v\right\|^{2} \leq C h_{k}^{2 s}\|v\|_{s, k}^{2} \leq C_{4} \lambda_{k}^{-s}\|v\|_{s, k}^{2}
$$

for some constant $C_{4}$, independent of $k$. By the discussion in Remark 1, we get that

$$
C_{4}^{-1} C_{1} J^{-1}\left(A_{h}^{s} v, v\right) \leq\left(B_{h}^{s} A_{h}^{s} v, A_{h}^{s} v\right) \leq C_{2} J\left(A_{h}^{s} v, v\right) \quad \forall v \in V_{h},
$$

and the condition number is bounded by $K\left(B_{h}^{s} A_{h}^{s}\right) \leq C J^{2}$.

5. Preconditioner when $s \in[-\mathbf{1}, \mathbf{0}]$. For $s \in[-1,0]$, the large eigenvalues of $A_{h}^{s}$ correspond to smooth functions. In a multilevel setting this means that neither relaxation nor coarse grid correction will reduce the oscillatory components of the error. As a consequence, we cannot expect a direct multigrid approach to work. Moreover, when $s<0$ the Löwner-Heinz and Jensen operator inequalities in (2.1) and (2.2) fail to hold, and the argument of section 4 is no longer valid. In this section, we will therefore investigate an alternative approach for constructing preconditioners.

We will base the preconditioner for $A_{h}^{s}$ when $s$ is negative on our previously defined preconditioners $B_{h}^{t}$ for $t \in[0,1]$ together with the multiplicative decomposition of $A_{h}$,

$$
A_{h}^{-s}=A_{h}^{-\frac{1+s}{2}} A_{h} A_{h}^{-\frac{1+s}{2}} .
$$

We have for every $u \in V_{h}$ and $t \in \mathbb{R}$ that

$$
\|u\|_{-s, h}=\left\|A_{h}^{-\frac{t+s}{2}} u\right\|_{t, h} .
$$

The specific form we will use below is

$$
\|u\|_{-\frac{1+s}{2}+\beta, h}=\left\|A_{h}^{-\frac{1+s}{2}} u\right\|_{\frac{1+s}{2}+\beta, h},
$$


which is valid for any $\beta \in \mathbb{R}$.

Replacing the leftmost and rightmost factors of the right-hand side in (5.1) with a spectrally equivalent preconditioner $B_{h}^{\frac{1+s}{2}}$ yields a symmetric positive-definite operator

$$
\tilde{B}_{h}^{s}:=B_{h}^{\frac{1+s}{2}} A_{h} B_{h}^{\frac{1+s}{2}}
$$

We want $\tilde{B}_{h}^{s}$ to be spectrally equivalent to $A_{h}^{-s}$. That is, there exist constants $C_{1}, C_{2}$ so that for every $u \in V_{h}$

$$
C_{1}\left(A_{h}^{s} u, u\right) \leq\left(\tilde{B}_{h}^{s} A_{h}^{s} u, A_{h}^{s} u\right) \leq C_{2}\left(A_{h}^{s} u, u\right)
$$

holds. By the definition of $\tilde{B}_{h}^{s}$,

$$
\left(\tilde{B}_{h}^{s} A_{h}^{s} u, A_{h}^{s} u\right)=\left(A_{h} B_{h}^{\frac{1+s}{2}} A_{h}^{s} u, B_{h}^{\frac{1+s}{2}} A_{h}^{s} u\right)=\left\|B_{h}^{\frac{1+s}{2}} A_{h}^{s} u\right\|_{1}^{2}
$$

and since $\left(A_{h}^{s} u, u\right)=\|u\|_{s, h}^{2}$, we see that the spectral equivalence in (5.4) is equivalent to

$$
C_{1}^{\frac{1}{2}}\|u\|_{s, h} \leq\left\|B_{h}^{\frac{1+s}{2}} A_{h}^{s} u\right\|_{1} \leq C_{2}^{\frac{1}{2}}\|u\|_{s, h} \quad \forall u \in V_{h} .
$$

Using the preconditioner described in section 4 , we have by the spectral equivalence established in Theorem 4.1 that there are constants $C_{1}, C_{2}>0$ so that

$$
C_{1}\|u\|_{-\frac{1+s}{2}, h} \leq\left\|B_{h}^{\frac{1+s}{2}} u\right\|_{\frac{1+s}{2}, h} \leq C_{2} J\|u\|_{-\frac{1+s}{2}, h}, \quad u \in V_{h} .
$$

We assume now some additional regularity on $B_{h}^{\frac{1+s}{2}}$, similar to (5.2). That is, for some $\beta$, we have the norm equivalence

$$
C_{1}\|u\|_{-\frac{1+s}{2}+\beta, h} \leq\left\|B_{h}^{\frac{1+s}{2}} u\right\|_{\frac{1+s}{2}+\beta, h} \leq C_{2} J\|u\|_{-\frac{1+s}{2}+\beta, h}
$$

In particular, with $\beta=\frac{1-s}{2} \in\left[\frac{1}{2}, 1\right]$, and replacing $u$ by $A_{h}^{s} u$ in (5.7), we recover (5.5) and the spectral equivalence (5.4). We note also that if we assume the additional regularity of (5.7), we can bound the condition number of $\tilde{B}_{h}^{s} A_{h}^{s}$ by

$$
K\left(\tilde{B}_{h}^{s} A_{h}^{s}\right) \leq K\left(B_{h}^{\frac{1+s}{2}} A_{h}^{\frac{1+s}{2}}\right)^{2}
$$

We remark that while (5.5) may be a nontrivial property to validate because $B_{h}$ is a discrete multigrid operator, similar conditions on the continuous differential operator are well established. That is, for $(-\Delta)^{-s}: H^{-s} \rightarrow H^{s}$ the regularity conditions that enable a decomposition $(-\Delta)^{-s}=(-\Delta)^{-(s+t)}(-\Delta)^{t}$ such that

$$
\left\|(-\Delta)^{-s}\right\|_{\mathcal{L}\left(H^{-s}, H^{s}\right)} \leq\left\|(-\Delta)^{-(s+t)}\right\|_{\mathcal{L}\left(H^{-s+t}, H^{s}\right)}\left\|(-\Delta)^{-s}\right\|_{\mathcal{L}\left(H^{-s}, H^{-s+t}\right)}
$$

are well described; cf. [31]. As such the regularity assumption (5.5) is a reasonable condition in the continuous setting, but the discrete setting is unclear. 
6. Implementational concerns. The discrete operators discussed so far are related to, but are not the same as, the matrices used in the implementation. In this section we will discuss how to implement these operators. We begin by discussing the matrix representation of the discrete fractional operators. We also refer the reader to [32] for more details. While the discrete fractional operators satisfy the group property $A_{h}^{s} A_{h}^{t}=A_{h}^{s+t}$, their matrix representations do not. In particular, for $t=-s$, $A_{h}^{s} A_{h}^{-s}=I_{h}$, and the finite element matrix representation of the identity is the mass matrix. Hence, in order to provide a precise description of the interpolation of the involved matrices, we let $\left\{\phi_{h}^{i}\right\}_{i=1}^{N_{h}}$ be the standard nodal basis for $V_{h}$, and we introduce the operators $\pi_{h}, \mu_{h}: V_{h} \rightarrow \mathbb{R}^{N_{h}}$, defined by

$$
\begin{aligned}
v & =\sum_{i=1}^{N_{h}}\left(\pi_{h} v\right)_{i} \phi_{h}^{i} \quad \text { and } \\
\left(\mu_{h} v\right)_{i} & =\left(v, \phi_{h}^{i}\right), \quad i=1, \ldots, N_{h} .
\end{aligned}
$$

Subsequently, we will refer to $\pi_{h} v$ and $\mu_{h} v$ as the primal and dual vector representations of $v$, respectively. The primal representation is sometimes called the nodal representation. We then have that

$$
\mu_{h}^{*}=\pi_{h}^{-1} \quad \text { and } \quad \pi_{h}^{*}=\mu_{h}^{-1} .
$$

To see this, take $v \in \mathbb{R}^{N_{h}}$ and $u \in V_{h}$. Then,

$$
\begin{aligned}
\left(\mu_{h}^{*} \mathrm{v}, u\right) & =\left(\mathrm{v}, \mu_{h} u\right)_{l^{2}} \\
& =\sum_{i=1}^{N_{h}} \mathrm{v}_{i}\left(u, \phi_{h}^{i}\right) \\
& =\left(u, \sum_{i=1}^{N_{h}} \mathrm{v}_{i} \phi_{h}^{i}\right) \\
& =\left(u, \pi_{h}^{-1} \mathrm{v}\right),
\end{aligned}
$$

where $(\cdot, \cdot)_{l^{2}}$ is the standard Euclidean inner product on $\mathbb{R}^{N_{h}}$. This proves the first identity in (6.2). The second identity is proven similarly.

Using these operators, the stiffness matrix can then be expressed as

$$
\mathrm{A}_{h}=\mu_{h} A_{h} \pi_{h}^{-1}, \quad \text { and } \quad\left(\mathrm{A}_{h}\right)_{i, j}=\left(A_{h} \phi_{h}^{j}, \phi_{h}^{i}\right), \quad 1 \leq i, j \leq N_{h},
$$

and the mass matrix is

$$
\mathrm{M}_{h}=\mu_{h} I_{h} \pi_{h}^{-1}=\mu_{h} \pi_{h}^{-1}, \quad \text { and } \quad\left(\mathrm{M}_{h}\right)_{i, j}=\left(\phi_{h}^{j}, \phi_{h}^{i}\right), \quad 1 \leq i, j \leq N_{h} .
$$

We see that for both the stiffness matrix and the mass matrix, a matrix-vector product takes primal vectors as input and returns dual vectors.

For the matrix realization of $A_{h}^{s}$, let $\left\{\left(\lambda_{i}, \mathbf{u}_{i}\right)\right\}_{i=1}^{N_{h}} \subset \mathbb{R} \times \mathbb{R}^{N_{h}}$ be the eigenpairs of the generalized eigenvalue problem

$$
\mathrm{A}_{h} \mathrm{u}_{i}=\lambda_{i} \mathrm{M}_{h} \mathrm{u}_{i}
$$

normalized so that $\mathbf{u}_{j}^{\top} \mathbf{M}_{h} \mathbf{u}_{i}=\delta_{i, j}$. Setting $\Lambda_{h}=\operatorname{diag}\left(\lambda_{1}, \ldots, \lambda_{N_{h}}\right)$, and $\mathbf{U}=\left[\mathbf{u}_{1}, \ldots, \mathbf{u}_{N_{h}}\right]$, we have that

$$
\mathrm{U}^{\top} \mathrm{M}_{h} \mathrm{U}=\mathrm{I} \quad \text { and } \quad \mathrm{U}^{\top} \mathrm{A}_{h} \mathrm{U}=\Lambda_{h}
$$


We then define

$$
\mathrm{A}_{h}^{s}=\left(\mathrm{M}_{h} \mathrm{U}\right) \Lambda_{h}^{s}\left(\mathrm{M}_{h} \mathrm{U}\right)^{\top}
$$

One can verify that the entries of $\mathrm{A}_{h}^{s}$ satisfy

$$
\left(\mathrm{A}_{h}^{s}\right)_{i, j}=\left(A_{h}^{s} \phi_{h}^{j}, \phi_{h}^{i}\right)
$$

in which case $\mathrm{A}_{h}^{s}=\mu_{h} A_{h}^{s} \pi_{h}^{-1}$. Using (6.3), we are also able to see that

$$
\left(\mathrm{A}_{h}^{s}\right)^{-1}=\mathrm{U} \Lambda_{h}^{-s} \mathrm{U}^{\top}=\pi_{h} A_{h}^{-s} \mu_{h}^{-1}
$$

making it the matrix realization of $A_{h}^{s}$ viewed as an operator from $X_{h}$ to $X_{h}^{\prime}$. However, the group properties mentioned above only make sense when we consider $A_{h}^{s}$ as operators on $X_{h}$. Thus, we see that for the matrices

$$
\pi_{h} A_{h}^{s} \pi_{h}^{-1}=\left(\pi_{h} \mu_{h}^{-1}\right) \mu_{h} A_{h}^{s} \pi_{h}^{-1}=\mathrm{M}_{h}^{-1} \mathrm{~A}_{h}^{s},
$$

the group properties are satisfied. This can also be verified using the definition of $\mathrm{A}_{h}^{s}$ in (6.4).

Since matrix-vector products involving $\mathrm{A}_{h}^{s}$ take primal vectors as input and return dual vectors, the matrix realization of $B_{h}^{s}$ should take dual vectors as input and return primal vectors. Then the product $\mathrm{B}_{h}^{s} \mathrm{~A}_{h}^{s}$ acts on primal vectors and is thus suitable for a Krylov subspace method. See also [32, section 6] and [10, section 15]. Therefore, we define

$$
\mathrm{B}_{h}^{s}=\pi_{h} B_{h}^{s} \mu_{h}^{-1}
$$

To see how $\mathrm{B}_{h}^{s}$ is implemented, we begin by supposing that $\operatorname{dim} V_{k}=N_{k}$ for $k=1, \ldots, J$. Let $\left\{\phi_{k}^{i}\right\}_{i=1}^{N_{k}}$ be bases for $V_{k}$, and we define operators $\pi_{k}, \mu_{k}: V_{k} \rightarrow \mathbb{R}^{N_{k}}$ analogously to (6.1). We then define mass and stiffness matrices on level $k$ as $\mathrm{M}_{k}=$ $\mu_{k} \pi_{k}^{-1}$ and $\mathrm{A}_{k}=\mu_{k} A_{k} \pi_{k}^{-1}$, respectively.

By assumption, for every $k, V_{k} \subset V_{h}$, and so there are matrices $\mathrm{I}_{k}: \mathbb{R}^{N_{k}} \rightarrow \mathbb{R}^{N_{h}}$ so that

$$
\phi_{k}^{i}=\sum_{j=1}^{N_{h}}\left(\mathrm{I}_{k}\right)_{i, j} \phi_{h}^{j}, \quad i=1, \ldots, N_{k} .
$$

In fact, $I_{k}$ is the matrix realization of the inclusion operator $I_{k}: V_{k} \rightarrow V_{h}$, i.e., $\mathrm{I}_{k}=\pi_{h} I_{k} \pi_{k}^{-1}$. Using that $Q_{k}=I_{k}^{*}$ and (6.2), we have that the transpose of $\mathrm{I}_{k}$ satisfies

$$
\begin{aligned}
\mathbf{I}_{k}^{\top} & =\left(\pi_{h} I_{k} \pi_{k}^{-1}\right)^{*} \\
& =\left(\pi_{k}^{-1}\right)^{*} Q_{k} \pi_{h}^{*} \\
& =\mu_{k} Q_{k} \mu_{h}^{-1} \\
& =: \mathbf{Q}_{k},
\end{aligned}
$$

which is the matrix realization of $Q_{k}$ in dual representation. Thus, for the matrix $\mathrm{B}_{h}^{s}$ 
we have that

$$
\begin{aligned}
\mathrm{B}_{h}^{s} & =\pi_{h} B_{h}^{s} \mu_{h}^{-1} \\
& =\sum_{k=1}^{J} \pi_{h} I_{k} R_{k}^{s} Q_{k} \mu_{h}^{-1} \\
& =\sum_{k=1}^{J}\left(\pi_{h} I_{k} \pi_{k}^{-1}\right)\left(\pi_{k} R_{k}^{s} \mu_{k}^{-1}\right)\left(\mu_{k} Q_{k} \mu_{h}^{-1}\right) \\
& =\sum_{k=1}^{J} \mathrm{Q}_{k}^{\top} \mathrm{R}_{k}^{s} \mathrm{Q}_{k},
\end{aligned}
$$

where we define $\mathrm{R}_{k}^{s}=\pi_{k} R_{k}^{s} \mu_{k}^{-1}$ as the matrix realization of $R_{k}^{s}$. We see that due to (6.5) $\mathrm{R}_{1}^{s}=\left(\mathrm{A}_{1}^{s}\right)^{-1}$. For $k \geq 2$ we define for $\nu \in \mathcal{N}_{k}$ operators $\pi_{k, \nu}, \mu_{k, \nu}: V_{k, \nu} \rightarrow$ $\mathbb{R}^{\operatorname{dim} V_{k, \nu}}$ and matrices $\mathrm{Q}_{k, \nu}: \mathbb{R}^{N_{k}} \rightarrow \mathbb{R}^{\operatorname{dim} V_{k, \nu}}$, similarly to the above. The matrix realization of $R_{k}^{s}$ then becomes

$$
\mathrm{R}_{k}^{s}=\sum_{\nu \in \mathcal{N}_{k}} \mathrm{Q}_{k, \nu}^{\top}\left(\mathrm{A}_{k, \nu}^{s}\right)^{-1} \mathrm{Q}_{k, \nu}
$$

Here, $\mathrm{A}_{k, \nu}^{s}=\mu_{k, \nu} A_{k, \nu}^{s} \pi_{k, \nu}^{-1}$. By (6.5), the implementation of $\mathrm{R}_{k}^{s}$ will require solving many small eigenvalue problems. In the particular case of continuous, piecewise linear finite element functions, and subdomains $\Omega_{k, \nu}$ as described in section 4, the subspaces $V_{k, \nu}$ are one-dimensional. The matrix $\mathrm{R}_{k}^{s}$ is then diagonal, with entries

$$
\left(\mathrm{R}_{k}^{s}\right)_{i, i}=\frac{1}{\left(\mathrm{M}_{k}\right)_{i, i}^{1-s}\left(\mathrm{~A}_{k}\right)_{i, i}^{s}}, \quad i=1, \ldots, N_{k} .
$$

That is, this is the smoother mentioned in the introduction.

Inserting (6.8) into (6.7), we get

$$
\mathrm{B}_{h}^{s}=\mathrm{Q}_{1}^{\top}\left(\mathrm{A}_{1}^{s}\right)^{-1} \mathrm{Q}_{1}+\sum_{k=2}^{J} \mathrm{Q}_{k}^{\top}\left(\sum_{\nu \in \mathcal{N}_{k}} \mathrm{Q}_{k, \nu}^{\top}\left(\mathrm{A}_{k, \nu}^{s}\right)^{-1} \mathrm{Q}_{k, \nu}\right) \mathrm{Q}_{k}
$$

We end this section by showing how to implement $\tilde{B}_{h}^{s}$ when $s \in[-1,0]$. In this case, the matrix realization of $\tilde{B}_{h}^{s}$ can be found from $\mathrm{B}_{h}^{\frac{1+s}{2}}$ and $\mathrm{A}_{h}$ by

$$
\begin{aligned}
\tilde{\mathrm{B}}_{h}^{s} & :=\pi_{h} \tilde{B}_{h}^{s} \mu_{h}^{-1} \\
& =\left(\pi_{h} B_{h}^{\frac{1+s}{2}} \mu_{h}^{-1}\right)\left(\mu_{h} A_{h} \pi_{h}^{-1}\right)\left(\pi_{h} B_{h}^{\frac{1+s}{2}} \mu_{h}^{-1}\right) \\
& =\mathrm{B}_{h}^{\frac{1+s}{2}} \mathrm{~A}_{h} \mathrm{~B}_{h}^{\frac{1+s}{2}} .
\end{aligned}
$$

That is, $\tilde{B}_{h}^{s}$ is implemented as an application of $B_{h^{\frac{1+s}{2}}}$, followed by a multiplication of the stiffness matrix and a second application of $B_{h}^{\frac{1+s}{2}}$.

7. Numerical experiments. In this section we present a series of numerical experiments that aim to validate the theoretical results we established in previous sections. We also present numerical results for the case when $s<0$, using $\tilde{B}_{h}^{s}$, defined in (5.3), as preconditioner. Specifically, in section 7.1 we solve

$$
A_{h}^{s} u=f
$$


TABLE 1

Numerical results for $(-\Delta)^{s}$ with nonnegative $s$. Table shows the number of preconditioned conjugate gradient iterations until reaching error tolerance $10^{-15}$. Estimated condition numbers are shown inside parentheses. $N$ is the number of elements on the finest mesh. $J=5$ in all tests.

\begin{tabular}{|c|c|c|c|c|c|}
\hline 3 & 32 & 64 & 128 & 256 & 512 \\
\hline 0.0 & $20(13.5)$ & $25(13.6)$ & $28(13.8)$ & $29(13.8)$ & $29(13.9)$ \\
\hline 0.1 & $18(8.7)$ & $21(8.9)$ & $23(8.9)$ & $24(8.9)$ & $24(8.9)$ \\
\hline 0.2 & $16(5.8)$ & $18(6.4)$ & $19(6.5)$ & $21(6.5)$ & $21(6.6)$ \\
\hline 0.3 & $14(4.2)$ & $15(4.7)$ & $17(4.9)$ & $18(5.0)$ & $18(5.0)$ \\
\hline 0.4 & $12(3.4)$ & $14(3.7)$ & $15(3.8)$ & $15(3.9)$ & $16(3.9)$ \\
\hline 0.5 & $11(2.9)$ & $12(3.0)$ & $13(3.1)$ & $13(3.1)$ & $14(3.2)$ \\
\hline 0.6 & $12(2.9)$ & $13(3.0)$ & $13(3.0)$ & $14(3.1)$ & $14(3.0)$ \\
\hline 0.7 & $12(3.0)$ & $13(3.0)$ & $14(3.1)$ & $14(3.1)$ & $14(3.1)$ \\
\hline 0.8 & $13(3.2)$ & $14(3.3)$ & $14(3.3)$ & $14(3.3)$ & $14(3.3)$ \\
\hline 0.9 & $14(3.5)$ & $15(3.6)$ & $15(3.6)$ & $15(3.6)$ & $15(3.6)$ \\
\hline 1.0 & $14(4.0)$ & $16(4.1)$ & $16(4.1)$ & $16(4.1)$ & $16(4.1)$ \\
\hline
\end{tabular}

using the preconditioned conjugate gradient method with $B_{h}^{s}$ defined in (4.5) as preconditioner. Here, the main motivation is to validate the $h$-independence of $K\left(B_{h}^{s} A_{h}^{s}\right)$ implied by Theorem 4.1. In section 7.2 , we consider a coupled multidomain problem where the weakly imposed continuity on the interface leads to a Lagrange multiplier in $H^{ \pm \frac{1}{2}}$

The numerical experiments are conducted using random initial guess. Convergence in the iterative methods used is reached when the relative preconditioned residual, i.e., $\frac{\left(B r_{k}, r_{k}\right)}{\left(B r_{0}, r_{0}\right)}$, where $r_{k}$ is the residual at the $k$ th iteration and $B$ is the preconditioner, is below a given tolerance.

7.1. Preconditioning the fractional Laplacian. In the first set of numerical experiments, we show the performance of the preconditioners $B_{h}^{s}$ and $\tilde{B}_{h}^{s}$, defined in (4.5) and (5.3), respectively, depending on the sign of $s$ for the $A_{h}^{s}$ inner product. That is, for a given $f_{h} \in V_{h}$, we solve the following: Find $u_{h} \in V_{h}$ such that

$$
\left(A_{h}^{s} u_{h}, v\right)=\left(f_{h}, v\right) \quad \forall v \in V_{h},
$$

where $s \in[-1,1]$. We take $\Omega=[0,1] \subset \mathbb{R}$, and $\mathcal{T}_{h}$ is a uniform partition of $\Omega$ consisting of $N=\frac{1}{h}$ elements. $V_{h}$ is then the space of continuous, piecewise linear functions relative to $\mathcal{T}_{h}$ that vanish on $\partial \Omega$. The matrix representation of $A_{h}^{s}$ we use here is provided by (6.4). This matrix is in general dense, and so matrix-vector multiplication will take $\mathcal{O}\left(N^{2}\right)$ operations. As such, the preconditioned iterative method will not be computationally optimal, but we stress that these experiments are designed only to validate the bounds on $K\left(B_{h}^{s} A_{h}^{s}\right)$.

We solve the linear system arising from (7.1) using preconditioned conjugate gradient, with $B_{h}^{s}$ as preconditioner if $s \geq 0$, and $\tilde{B}_{h}^{s}$ if $s<0$. For $s \geq 0$, iteration counts and estimated condition numbers can be viewed in Table 1. From these results we see that both the iteration counts and condition numbers stay uniformly bounded for each $s$.

The analogous results for $s \leq 0$ can be seen in Table 2. Here the situation is slightly more complicated. For each $s$, the iteration counts and condition numbers seem to increase for small $N$ (large $h$ ) but ultimately stay bounded when $N$ is increased. Worth noting is that the bound (5.8) is relatively sharp. For instance, for $s=-1$, the preconditioner $\tilde{B}_{h}^{s}$ does two applications of $B_{h}^{0}$ and has estimated condition numbers around 193. By Table $1, K\left(B_{h}^{0} A_{h}^{0}\right) \approx 13.8$, and so 
TABLE 2

Numerical results for $(-\Delta)^{s}$ with negative $s$. Table shows the number of preconditioned conjugate gradient iterations until reaching error tolerance $10^{-15}$. Estimated condition numbers are shown inside parentheses. $N$ is the number of elements on the finest mesh. $J=5$ in all tests.

\begin{tabular}{l|lllll}
\multicolumn{1}{l|}{$N$} & 32 & 64 & 128 & 256 & 512 \\
\hline-1.0 & $32(184.4)$ & $47(192.4)$ & $56(192.7)$ & $64(193.8)$ & $62(191.2)$ \\
-0.9 & $28(119.0)$ & $43(118.9)$ & $50(120.5)$ & $54(120.7)$ & $55(119.9)$ \\
-0.8 & $26(78.3)$ & $37(82.6)$ & $46(84.5)$ & $48(83.8)$ & $49(83.9)$ \\
-0.7 & $25(53.0)$ & $33(60.1)$ & $40(61.9)$ & $42(62.1)$ & $45(61.5)$ \\
-0.6 & $24(36.9)$ & $31(43.8)$ & $35(45.8)$ & $38(46.2)$ & $41(46.2)$ \\
-0.5 & $22(26.8)$ & $25(31.9)$ & $30(34.3)$ & $34(34.9)$ & $38(35.1)$ \\
-0.4 & $20(20.4)$ & $24(24.8)$ & $28(26.5)$ & $32(27.0)$ & $37(27.1)$ \\
-0.3 & $17(16.1)$ & $21(19.3)$ & $27(20.7)$ & $30(21.1)$ & $34(21.1)$ \\
-0.2 & $17(13.1)$ & $21(15.3)$ & $25(16.4)$ & $29(16.7)$ & $32(16.7)$ \\
-0.1 & $16(11.0)$ & $20(12.4)$ & $23(13.2)$ & $27(13.5)$ & $29(13.5)$ \\
0.0 & $14(9.4)$ & $17(10.4)$ & $20(11.0)$ & $24(11.2)$ & $27(11.1)$
\end{tabular}

$K\left(\tilde{B}^{-1} A_{h}^{-1}\right) \approx K\left(B_{h}^{0} A_{h}^{0}\right)^{2}$. Similar relations holds for other values of $s \leq 0$.

7.2. Multidomain preconditioning. In this section we apply the multilevel algorithm (4.5) to construct mesh independent preconditioners for a coupled multidomain problem originating from a geometrically accurate model of electric signal propagation in cardiac tissue, the Extracellular-Membrane-Intracellular (EMI) model [35]. We remark that the EMI model is simple in the sense that it is a single-physics problem where two elliptic equations are coupled. However, the interface problems encountered here are identical to those found in multiphysics applications, e.g., the coupled Darcy-Stokes system [29] or the Stokes-Biot system [2].

Let $\Omega \subset \mathbb{R}^{2}$ be a bounded domain decomposed into two nonoverlapping subdomains $\Omega_{1}, \Omega_{2}$ with a common interface $\Gamma=\partial \Omega_{1} \bigcap \partial \Omega_{2}$ forming a closed curve. Motivated by the application, the subdomain $\Omega_{1}$ is designated as the exterior domain, i.e., $\partial \Omega_{2} \bigcap \partial \Omega=\emptyset$. With $\epsilon>0$ and $n$ the outer normal of the exterior domain we now aim to solve

$$
\begin{aligned}
& u_{1}-\Delta u_{1}=f_{1}, \quad x \in \Omega_{1}, \\
& u_{2}-\Delta u_{2}=f_{2}, \quad x \in \Omega_{2}, \\
& n \cdot \nabla u_{1}-n \cdot \nabla u_{2}=0, \quad x \in \Gamma, \\
& \epsilon\left(u_{1}-u_{2}\right)+n \cdot \nabla u_{1}=g, \quad x \in \Gamma \text {. }
\end{aligned}
$$

The choice of boundary conditions for (7.2) shall be discussed shortly. We remark that in the EMI model the parameter $\epsilon$ plays a role of inverse time step, and thus algorithms robust with respect to the parameter are of interest. However, here the system will be considered only for a fixed choice of the parameter.

Considering (7.2) with homogeneous Neumann boundary conditions $n \cdot \nabla u_{1}=0$ on $\partial \Omega$ and letting $W_{1}=H^{1}\left(\Omega_{1}\right) \times H^{1}\left(\Omega_{2}\right) \times\left(H^{-1 / 2}(\Gamma) \bigcap \epsilon^{-1 / 2} L^{2}(\Gamma)\right)$, the variational formulation of (7.2) defines an operator $\mathcal{A}_{1}: W_{1} \rightarrow W_{1}^{\prime}$,

$$
\mathcal{A}_{1}=\left(\begin{array}{ccc}
I-\Delta & 0 & T_{1}^{*} \\
0 & I-\Delta & -T_{2}^{*} \\
T_{1} & -T_{2} & -\epsilon^{-1} I
\end{array}\right),
$$

where $T_{i}, T_{i} v=\left.v\right|_{\Gamma}$ for $v \in C\left(\bar{\Omega}_{i}\right), i=1,2$, are the trace operators on $H^{1}\left(\Omega_{1}\right)$ and $H^{1}\left(\Omega_{2}\right)$, respectively. Tveito et al. [35] further discuss a mixed formulation of the 
system (7.2) where additional unknowns $\sigma_{i}=-\nabla u_{i}, i=1,2$, are introduced. If homogeneous Dirichlet boundary conditions $u_{1}=0$ on $\partial \Omega$ are assumed, the mixed formulation leads to operator $\mathcal{A}_{2}: W_{2} \rightarrow W_{2}^{\prime}$,

$$
\mathcal{A}_{2}=\left(\begin{array}{ccc}
I & \nabla & T^{*} \\
-\nabla \cdot & -I & 0 \\
T & 0 & -\epsilon I
\end{array}\right)
$$

with $W_{2}=H(\operatorname{div}, \Omega) \times L^{2}(\Omega) \times\left(H^{1 / 2}(\Gamma) \bigcap \epsilon^{1 / 2} L^{2}(\Gamma)\right)$ and $T$ the normal trace operator $T \sigma=\left.\sigma\right|_{\Gamma} \cdot n$ for $v \in[C(\Omega)]^{2}$. We remark that operators $\mathcal{A}_{1}$ and $\mathcal{A}_{2}$ also arise naturally in the analysis of nonoverlapping domain decomposition methods for second order elliptic problems in the primal [36] and mixed [18] formulations, respectively.

Assuming that the operators $\mathcal{A}_{1}$ and $\mathcal{A}_{2}$ are isomorphisms on their respective spaces, ${ }^{1}$ the preconditioners can be established within the framework of operator preconditioning [32]. In particular, the Riesz map preconditioner for (7.3) is

$$
\mathcal{B}_{1}=\left(\begin{array}{lll}
I-\Delta & & \\
& I-\Delta & \\
& & \epsilon^{-1} I+(-\Delta+I)^{-1 / 2}
\end{array}\right)^{-1},
$$

while (7.4) will be preconditioned by

$$
\mathcal{B}_{2}=\left(\begin{array}{lll}
I-\nabla \nabla \cdot & & \\
& I & \\
& & \epsilon I+(-\Delta+I)^{1 / 2}
\end{array}\right)^{-1} .
$$

Note that the operator sums in $\mathcal{B}_{1}, \mathcal{B}_{2}$ are due to the fact that the interface spaces are intersection spaces [7].

In order to simplify the setting and focus only on the fractional operators in the preconditioners, we remove the parameter dependence from the problems by setting $\epsilon=\infty$ in (7.3), (7.5) and similarly $\epsilon=0$ for (7.4), (7.6). In turn, the interface spaces reduce to $H^{-1 / 2}(\Gamma)$ and $H^{1 / 2}(\Gamma)$, respectively, and the multilevel algorithm is directly applicable to the related interface problems which now involve the operator $I-\Delta$; cf. the Laplacian operator in the previous sections.

Robustness of $\mathcal{B}_{1}, \mathcal{B}_{2}$ and in particular the fractional Sobolev space preconditioner are finally demonstrated by observing the iteration counts of the preconditioned MinRes method. In the experiments we let $\Omega=[0,1]^{2}$ and $\Omega_{2}=[0.25,0.75]^{2}$. The finite element discretization of $W_{1}$ uses continuous linear Lagrange elements $\left(\mathrm{P}_{1}\right)$. For $W_{2}$ two different discretizations are considered: $\mathrm{BDM}_{1}-\mathrm{P}_{0}-\mathrm{P}_{1}$ and $\mathrm{RT}_{1}-\mathrm{P}_{0}-\mathrm{P}_{0}$. That is, the first subspace of $W_{2}$ is constructed from linear Brezzi-Douglas-Marini element $\left(\mathrm{BDM}_{1}\right)$ or the lowest order Raviart-Thomas element $\left(\mathrm{RT}_{1}\right)$, while the remaining subspaces use piecewise constant and piecewise linear Lagrange elements, respectively. Let us note that with the $\mathrm{RT}_{1}-\mathrm{P}_{0}-\mathrm{P}_{0}$ element the discretization is nonconforming owing to the piecewise constant space for the multiplier. Moreover, the fractional multigrid algorithm is then applied outside the setting used in the analysis of section 4 . We remark that in this case the multigrid preconditioner uses the discrete operator

$$
\left(A_{h} u, w\right)=(u, w)+\sum_{\nu \in \mathcal{N}}\{\{h\}\}_{\nu}^{-1} \llbracket u \rrbracket_{\nu} \llbracket w \rrbracket_{\nu}, \quad u, w \in V_{h}
$$

\footnotetext{
${ }^{1}$ The proof of this result as well as stable finite element discretization of the problem are the subject of current work and will be reported elsewhere. We remark that operator $\mathcal{A}_{1}$ in the limit case $\epsilon=\infty$ has been studied in [28] in the context of the mortar finite element method.
} 
TABLE 3

Number of MinRes iterations for the operator $B_{1} \mathcal{A}_{1}$ and $\epsilon=10^{15}$ using multilevel algorithm with $J$ levels as a preconditioner for $(-\Delta+I)^{-1 / 2}$. Realizing the fractional operator with spectral decomposition leads to iteration counts in the last column.

\begin{tabular}{c|c|c||ccc|c}
\hline \multirow{2}{*}{$h$} & \multirow{2}{*}{$\operatorname{dim} W_{h}$} & \multirow{2}{*}{ \# cells } & \multicolumn{3}{|c|}{ \#MG } & \multirow{2}{*}{ \# Eig } \\
\cline { 4 - 5 } & & & $J=2$ & $J=3$ & $J=4$ & \\
\hline $2.21 \mathrm{E}-02$ & 4481 & 128 & 67 & 93 & 103 & 36 \\
$1.10 \mathrm{E}-02$ & 17153 & 256 & 68 & 92 & 111 & 35 \\
$5.52 \mathrm{E}-03$ & 67073 & 512 & 66 & 90 & 112 & 35 \\
$2.76 \mathrm{E}-03$ & 265217 & 1024 & 64 & 90 & 112 & 34 \\
$1.38 \mathrm{E}-03$ & 1054721 & 2048 & 64 & 88 & 108 & 33 \\
\hline
\end{tabular}

TABLE 4

Number of MinRes iterations for the operator $B_{2} \mathcal{A}_{2}$ and $\epsilon=10^{-15}$ using multilevel algorithm with $J$ levels as a preconditioner for $(-\Delta+I)^{1 / 2}$. Two different finite element discretizations are considered. Realizing the fractional operator with spectral decomposition leads to iteration counts in the \#Eig column.

\begin{tabular}{|c|c|c|c|c|c|c|c|c|c|c|c|}
\hline \multirow{3}{*}{$h$} & \multirow{3}{*}{$\#$ cells ${ }_{\Gamma}$} & \multicolumn{5}{|c|}{$\mathrm{BDM}_{1}-\mathrm{P}_{0}-\mathrm{P}_{1}$} & \multicolumn{5}{|c|}{$\mathrm{RT}_{1}-\mathrm{P}_{0}-\mathrm{P}_{0}$} \\
\hline & & \multirow{2}{*}{$\operatorname{dim} W_{h}$} & \multicolumn{3}{|c|}{ \#MG } & \multirow{2}{*}{ \#Eig } & \multirow{2}{*}{$\operatorname{dim} W_{h}$} & \multicolumn{3}{|c|}{ \#MG } & \multirow{2}{*}{ \#Eig } \\
\hline & & & $J=2$ & $J=3$ & $J=4$ & & & $J=2$ & $J=3$ & $J=4$ & \\
\hline $2.21 \mathrm{E}-02$ & 128 & 33152 & 25 & 27 & 28 & 22 & 20736 & 27 & 28 & 27 & 22 \\
\hline $1.10 \mathrm{E}-02$ & 256 & 131840 & 25 & 27 & 29 & 22 & 82432 & 27 & 32 & 32 & 22 \\
\hline $5.52 \mathrm{E}-03$ & 512 & 525824 & 23 & 27 & 27 & 22 & 328704 & 27 & 33 & 36 & 22 \\
\hline $2.76 \mathrm{E}-03$ & 1024 & 2100224 & 22 & 27 & 29 & 22 & 1312768 & 27 & 33 & 40 & 22 \\
\hline $1.38 \mathrm{E}-03$ & 2048 & 8394752 & 22 & 25 & 29 & 22 & 5246976 & 25 & 35 & 40 & 22 \\
\hline
\end{tabular}

Here $\mathcal{N}$ is a set of all the vertices of the mesh and $\{\{u\}\}_{\nu}=\frac{1}{2}\left(\left.u\right|_{K^{+}}+\left.u\right|_{K^{-}}\right), \llbracket u \rrbracket_{\nu}=$ $\left.u\right|_{K^{+}}-\left.u\right|_{K^{-}}$are the average and the jump values computed from the two ${ }^{2}$ cells $K^{ \pm}$ connected to the vertex $\nu$. Moreover, $\operatorname{dim} V_{k, \nu}=2$ so that the eigenvalue problems needing to be solved by the smoother 4.2 use 2 by 2 matrices. Compare this to the case of one-dimensional problems due to continuous, piecewise linear elements in section 6 .

The discrete preconditioners shall use off-the-shelf methods for the first two blocks. More specifically, a single V-cycle of algebraic multigrid is used for $\mathcal{B}_{1}$ while for $\mathcal{B}_{2}$ the action is computed exactly by a direct solver. The final block of the preconditioners is realized by the proposed multilevel preconditioner with a different number of levels $J=2,3,4$.

The number of MinRes iterations is shown in Tables 3 and 4. Here, the iterations were started from a random initial vector and terminated once the relative preconditioned residual norm was less then $10^{-8}$ in magnitude. For both $\mathcal{B}_{1}$ and $\mathcal{B}_{2}$ the iterations are bounded in the discretization parameter. The tables further list iteration counts for preconditioners where the fractional operators were realized in terms of spectral decomposition. As expected from the theory and experiments for the Laplace problem, the difference in iteration counts between the multilevel realization and spectral realization is larger for $\mathcal{B}_{1}$ than it is for $\mathcal{B}_{2}$.

\section{REFERENCES}

[1] S. Aland, S. Egerer, J. Lowengrub, And A. Voigt, Diffuse interface models of locally inextensible vesicles in a viscous fluid, J. Comput. Phys., 277 (2014), pp. 32-47.

[2] I. Ambartsumyan, E. Khattatov, I. Yotov, and P. Zunino, A Lagrange multiplier method for a Stokes-Biot fluid-poroelastic structure interaction model, Numer. Math., 140 (2018), pp. 513-553.

\footnotetext{
${ }^{2}$ Recall that the interface $\Gamma$ is here taken as a simple closed curve.
} 
[3] M. Arioli, D. Kourounis, And D. Loghin, Discrete fractional Sobolev norms for domain decomposition preconditioning, IMA J. Numer. Anal., 33 (2013), pp. 318-342.

[4] M. Arioli And D. Loghin, Discrete interpolation norms with applications, SIAM J. Numer. Anal., 47 (2009), pp. 2924-2951, https://doi.org/10.1137/080729360.

[5] I. BABUŠKA, The finite element method with Lagrangian multipliers, Numer. Math., 20 (1972/73), pp. 179-192.

[6] A. V. BALAKRISHNAN, Fractional powers of closed operators and the semigroups generated by them, Pacific J. Math., 10 (1960), pp. 419-437.

[7] J. Bergh AND J. LÖFströM, Interpolation Spaces. An Introduction, Grundlehren Math. Wiss. 223, Springer-Verlag, Berlin, 1976

[8] S. Bertoluzza, V. Chabannes, C. Prud'homme, and M. Szopos, Boundary conditions involving pressure for the Stokes problem and applications in computational hemodynamics, Comput. Methods Appl. Mech. Engrg., 322 (2017), pp. 58-80.

[9] A. Bonito AND J. E. PASCIAK, Numerical approximation of fractional powers of elliptic operators, Math. Comp., 84 (2015), pp. 2083-2110.

[10] J. H. Bramble, Multigrid Methods, Pitman Res. Notes Math. Ser. 294, Longman, Harlow, UK, 1993.

[11] J. H. Bramble and J. E. Pasciak, New convergence estimates for multigrid algorithms, Math. Comp., 49 (1987), pp. 311-329.

[12] J. H. Bramble, J. E. Pasciak, and P. S. Vassilevski, Computational scales of Sobolev norms with application to preconditioning, Math. Comp., 69 (2000), pp. 463-480.

[13] J. H. Bramble, J. E. Pasciak, J. P. WAng, And J. Xu, Convergence estimates for product iterative methods with applications to domain decomposition, Math. Comp., 57 (1991), pp. 1-21.

[14] J. H. Bramble, J. E. Pasciak, and J. Xu, Parallel multilevel preconditioners, Math. Comp., 55 (1990), pp. 1-22.

[15] J. H. Bramble, J. E. PASciak, And J. Xu, The analysis of multigrid algorithms with nonnested spaces or noninherited quadratic forms, Math. Comp., 56 (1991), pp. 1-34.

[16] K. Burrage, N. Hale, AND D. KAY, An efficient implicit FEM scheme for fractional-inspace reaction-diffusion equations, SIAM J. Sci. Comput., 34 (2012), pp. A2145-A2172, https://doi.org/10.1137/110847007.

[17] S. Court And M. Fournié, A fictitious domain finite element method for simulations of fluidstructure interactions: The Navier-Stokes equations coupled with a moving solid, J. Fluids Struct., 55 (2015), pp. 398-408.

[18] L. C. Cowsar, J. MAndel, And M. F. Wheeler, Balancing domain decomposition for mixed finite elements, Math. Comp., 64 (1995), pp. 989-1015.

[19] N. Hale, N. J. Higham, and L. N. Trefethen, Computing $\mathbf{A}^{\alpha}, \log (\mathbf{A})$, and related matrix functions by contour integrals, SIAM J. Numer. Anal., 46 (2008), pp. 2505-2523, https: //doi.org/10.1137/070700607.

[20] F. Hansen AND G. KJæRGÅRD Pedersen, Jensen's inequality for operators and Löwner's theorem, Math. Ann., 258 (1982), pp. 229-241.

[21] F. Hansen And G. K. Pedersen, Jensen's operator inequality, Bull. London Math. Soc., 35 (2003), pp. 553-564.

[22] S. Harizanov, R. Lazarov, P. Marinov, S. Margenov, and Y. Vutov, Optimal Solvers for Linear Systems with Fractional Powers of Sparse SPD Matrices, preprint, https://arxiv. org/abs/1612.04846, 2016.

[23] T. KATO, Notes on some inequalities for linear operators, Math. Ann., 125 (1952), pp. $208-212$.

[24] L. Knizhnerman And V. Simoncini, A new investigation of the extended Krylov subspace method for matrix function evaluations, Numer. Linear Algebra Appl., 17 (2010), pp. 615638.

[25] M. Kočvara, D. Loghin, And J. Turner, Constraint interface preconditioning for topology optimization problems, SIAM J. Sci. Comput., 38 (2016), pp. A128-A145, https://doi.org/ $10.1137 / 140980387$.

[26] M. Kuchta, K.-A. Mardal, and M. Mortensen, Preconditioning Trace Coupled 3d-1d Systems Using Fractional Laplacian, preprint, https://arxiv.org/abs/1612.03574, 2016.

[27] M. Kuchta, M. Nordaas, J. C. G. Verschaeve, M. Mortensen, and K.-A. Mardal, Preconditioners for saddle point systems with trace constraints coupling $2 D$ and $1 D$ domains, SIAM J. Sci. Comput., 38 (2016), pp. B962-B987, https://doi.org/10.1137/15M1052822.

[28] B. P. Lamichhane and B. I. Wohlmuth, Mortar finite elements for interface problems, Computing, 72 (2004), pp. 333-348.

[29] W. J. LAYton, F. SchiEwECK, AND I. Yotov, Coupling fluid flow with porous media flow, SIAM J. Numer. Anal., 40 (2003), pp. 2195-2218, https://doi.org/10.1137/ 
S0036142901392766.

[30] J.-L. Lions and E. Magenes, Non-Homogeneous Boundary Value Problems and Applications. Vol. I, translated from the French by P. Kenneth, Grundlehren Math. Wiss. 181, SpringerVerlag, New York, 1972

[31] A. Lischke, G. Pang, M. Gulian, F. Song, C. Glusa, X. Zheng, Z. Mao, W. Cai, M. M. Meerschaert, M. Ainsworth, and G. E. Karniadakis, What Is the Fractional Laplacian?, preprint, https://arxiv.org/abs/1801.09767, 2018.

[32] K.-A. Mardal and R. Winther, Preconditioning discretizations of systems of partial differential equations, Numer. Linear Algebra Appl., 18 (2011), pp. 1-40.

[33] P. Oswald, Multilevel norms for $H^{-1 / 2}$, Computing, 61 (1998), pp. 235-255.

[34] R. Stevenson, Stable three-point wavelet bases on general meshes, Numer. Math., 80 (1998), pp. $131-158$.

[35] A. Tveito, K. H. Jeger, M. Kuchta, K.-A. Mardal, and M. E. Rognes, A cell-based framework for numerical modeling of electrical conduction in cardiac tissue, Frontiers Phys., 5 (2017), 48.

[36] B. I. Wohlmuth, A mortar finite element method using dual spaces for the Lagrange multiplier, SIAM J. Numer. Anal., 38 (2000), pp. 989-1012, https://doi.org/10.1137/ S0036142999350929.

[37] J. Xu, Iterative methods by space decomposition and subspace correction, SIAM Rev., 34 (1992), pp. 581-613, https://doi.org/10.1137/1034116.

[38] Q. YAng, I. Turner, F. Liu, And M. ILIĆ, Novel numerical methods for solving the timespace fractional diffusion equation in two dimensions, SIAM J. Sci. Comput., 33 (2011), pp. 1159-1180, https://doi.org/10.1137/100800634. 